\title{
A seismic approach to testing different formation channels of subdwarf B stars
}

\author{
Haili Hu ${ }^{1,2}$, M.-A. Dupret ${ }^{3}$, C. Aerts ${ }^{1,2}$, G. Nelemans ${ }^{1}$, S. D. Kawaler ${ }^{4}$, A. Miglio ${ }^{5}$, J. Montalban ${ }^{5}$, and R. Scuflaire \\ 1 Department of Astrophysics, IMAPP, Radboud University Nijmegen, PO Box 9010, 6500 GL, Nijmegen, The Netherlands \\ e-mail: hailihu@astro.ru.nl \\ 2 Institute of Astronomy, Katholieke Universiteit Leuven, Celestijnenlaan 200D, 3001 Leuven, Belgium \\ 3 Observatoire de Paris, LESIA, 5 place Jules Janssen, 92195 Meudon Principal Cedex, France \\ 4 Department of Physics and Astronomy, Iowa State Univeristy, Ames, IA 50014, USA \\ 5 Institute d'Astrophysique et Géophysique, Université de Liège, allée du 6 Août, 17, 4000 Liège, Belgium
}

Received 21 May 2008 / Accepted 22 July 2008

\begin{abstract}
Context. There are many unknowns in the formation of subdwarf B stars. Different formation channels are considered to be possible and to lead to a variety of helium-burning subdwarfs. All seismic models to date, however, assume that a subdwarf B star is a posthelium-flash-core surrounded by a thin inert layer of hydrogen.

Aims. We examine an alternative formation channel, in which the subdwarf B star originates from a massive $\left(>\sim 2 M_{\odot}\right)$ red giant with a non-degenerate helium-core. Although these subdwarfs may evolve through the same region of the $\log g-T_{\text {eff }}$ diagram as the canonical post-flash subdwarfs, their interior structure is rather different. We examine how this difference affects their pulsation modes and whether it can be observed.

Methods. Using detailed stellar evolution calculations we construct subdwarf B models from both formation channels. The iron accumulation in the driving region due to diffusion, which causes the excitation of the modes, is approximated by a Gaussian function. The pulsation modes and frequencies are calculated with a non-adiabatic pulsation code.

Results. A detailed comparison of two subdwarf B models from different channels, but with the same $\log g$ and $T_{\text {eff }}$, shows that their mode excitation is different. The excited frequencies are lower for the post-flash than for the post-non-degenerate subdwarf B star. This is mainly due to the differing chemical composition of the stellar envelope. A more general comparison between two grids of models shows that the excited frequencies of most post-non-degenerate subdwarfs cannot be well-matched with the frequencies of post-flash subdwarfs. In the rare event that an acceptable seismic match is found, additional information, such as mode identification and $\log g$ and $T_{\text {eff }}$ determinations, allows us to distinguish between the two formation channels.
\end{abstract}

Key words. stars: subdwarfs - stars: evolution - stars: oscillations - methods: numerical

\section{Introduction}

Commonly, subdwarf B (sdB) stars are identified as extreme horizontal branch (EHB) stars, and they are believed to be post-Hecore-flash products with core masses $\sim 0.5 M_{\odot}$ surrounded by a very thin inert H-envelope (Heber 1986; Saffer et al. 1994). From a single stellar evolution point of view, this can be explained by enhanced mass loss of stars close to He-ignition with very lightly bound envelopes (D'Cruz et al. 1996), i.e. stars with degenerate cores near the tip of the red giant branch (RGB). However, as they are frequently observed in binaries (e.g. Allard et al. 1994; Morales-Rueda et al. 2006), binary interactions most likely play an important role in their formation. Han et al. (2002) explored the main binary evolution channels that can produce sdB stars: common-envelope ejection (CEE), stable Roche lobe overflow (RLOF), and helium white dwarf mergers. They found that the sdB mass distribution may be much broader than previously thought, $0.3-0.8 M_{\odot}$ instead of $0.4-0.5 M_{\odot}$. The sdB stars with non-canonical masses follow from mergers or massive ( $>\sim 2 M_{\odot}$ ) progenitors that ignite helium quiescently, where the latter can be a subchannel of either the CEE channel or the stable RLOF channel. Binary population synthesis shows that the massive progenitors do not contribute significantly to the
sdB population (Han et al. 2003). But one should keep in mind that it is asumed in such studies that $\mathrm{CE}$ evolution is described by the $\alpha$ formalism, i.e. that the CE ejection is driven by the orbital energy. Because the physics of the CE phase is poorly understood, other scenarios should not be excluded a priori. For example, the $\gamma$-formalism proposed by Nelemans et al. (2000), based on the angular momentum equation rather than the energy equation, provides an alternative description. In this case, the massive red giants cannot be ruled out as possible progenitors of post-CE sdB stars (Hu et al. 2007). We therefore want to explore the possibility of this neglected class of progenitors in a different manner, by using the seismic properties that have been observed in some sdB stars.

Although the post-flash and the post-non-degenerate sdB stars can appear in the same log $g-T_{\text {eff }}$ region, their interior structure is quite different. In particular, the chemical composition profiles differ greatly depending on whether helium ignited in a flash or quiescently. For example, the canonical post-Heflash sdB star has a very narrow $\mathrm{He}-\mathrm{H}$ transition zone, while the $\mathrm{sdB}$ star created from a more massive progentior has a much broader H-profile. This is a direct result of the differing chemical compositions between low-mass and high-mass stars on the RGB, owing to the different convective regions during the 
main-sequence and RGB evolution. We examine whether this difference in the interior structure will result in observable differences in the pulsation modes.

The sdB pulsators consist of two classes, the short-period variable EC 14026 stars (Kilkenny et al. 1997), and the longperiod variable PG 1716 stars (Green et al. 2003). The rapid oscillations in EC 14026 stars are interpreted in terms of low-order $p$-modes (Charpinet et al. 1996), driven by the $\kappa$-mechanism operating in the iron opacity bump. The same mechanism has been shown to excite long-period, high-order $g$-modes in the cooler models (Fontaine et al. 2003). The local iron enhancement necessary in the driving region around $\log T \approx 5.3$ is due to the competing diffusion processes of radiative levitation and gravitational settling. It is well-known that the opacities play an important role in the study of the pulsations. Seaton \& Badnell (2004) showed that the iron opacity bump is situated at slightly higher temperatures using OP opacities (Seaton et al. 1994; Badnell \& Seaton 2003) compared with OPAL opacities (Iglesias \& Rogers 1996). Jeffery \& Saio (2006) found that, using OP opacities and nickel enhancement in addition to iron, the theoretical instability strip of $g$-mode sdB oscillators is more consistent with observations. For our purposes it is sufficient to use OPAL opacities and iron enhancement, since we are interested in the relative differences between two types of sdB stars. We acknowledge the importance of including the effect of OP opacities and nickel enhancement in further detailed studies.

The details of the computations are given in Sect. 2. The results are presented in Sect. 3. In Sect. 3.3, we compare the detailed physical characteristics of two reference models with different formation histories. In Sect. 3.4 we compare the frequency characteristics globally between two grids of models. The results and conclusions are discussed in Sect. 4.

\section{Computations}

\subsection{The evolution calculations}

We constructed sdB structure models with the stellar evolution code developed by Eggleton (1971, 1972, 1973), Eggleton et al. (1973), and updated by Han et al. (1994) and Pols et al. (1995, 1998). The updated version of the code uses an equation of state that includes pressure ionization and Coulomb interaction, nuclear reaction rates from Caughlan et al. (1985) and Caughlan \& Fowler (1988), and neutrino loss rates from Itoh et al. (1989, 1992). Both convective and semi-convective mixing are treated as diffusion processes. It is assumed that mixing occurs in regions where

$\nabla_{\mathrm{rad}}>\nabla_{\mathrm{ad}}-\delta_{\mathrm{ov}} /\left(2.5+20 \beta+16 \beta^{2}\right)$,

where $\beta$ is the ratio of radiation pressure to gas pressure and $\delta_{\mathrm{ov}}$ is the overshooting parameter. Schröder et al. (1997) showed that $\delta_{\mathrm{ov}}=0.12$ gives the best fit to observations of $\zeta$ Aurigae binaries, which corresponds to an overshooting length of $\sim 0.25 H_{\mathrm{p}}$. For our comparative study, it suffices to adopt $\delta_{\text {ov }}=0.12$, but keep in mind that core overshooting can in fact also be probed by asteroseismology, e.g. Aerts et al. (2003).

We evolved stars assuming a chemical composition of $X=0.70$ and $Z=0.02$. We used a mixing-length parameter (the ratio of the mixing-length to the local pressure scaleheight) of $\alpha=l / H_{\mathrm{p}}=2.0$. If not mentioned otherwise, the opacity tables were constructed by combining the OPAL opacities (Iglesias \& Rogers 1996) with the conductive opacities (Hubbard \& Lampe 1969; Canuto 1970; Iben 1975), as implemented in the Eggleton code by Eldridge \& Tout (2004).
We started by evolving zero-age main-sequence (ZAMS) models in the range $1-3 M_{\odot}$ to the tip of the RGB, adopting a Reimer's mass loss rate (Reimers 1975),

$\dot{M}_{\text {wind }}=4 \times 10^{-13} \eta \frac{\left(R / R_{\odot}\right)\left(L / L_{\odot}\right)}{\left(M / M_{\odot}\right)}\left[M_{\odot} \mathrm{yr}^{-1}\right]$,

with an efficiency of $\eta=0.4$ (Iben \& Renzini 1983; Carraro et al. 1996). For simplicity, we did not include mass-loss on the EHB. Unglaub \& Bues (2001) showed that, if the observed chemical abundances are the result of the combined effects of diffusion and mass loss, the sdB mass-loss rate should be in the range $10^{-14} \leq \dot{M}\left(M_{\odot} \mathrm{yr}^{-1}\right) \leq 10^{-12}$. This is consistent with the rates found by Vink \& Cassisi (2002) for radiation-driven wind models. They also showed that these rates are too low to have a direct effect on the sdB evolution.

At the RGB tip, we removed the envelope, while keeping the chemical compositions fixed. Thus, we assume that the mass transfer happens on a much shorter timescale than the nuclear timescale. This is a reasonable assumption for $\mathrm{sdB}$ stars in shortperiod binaries formed by $\mathrm{CE}$ ejection, which is the majority of the observed sdB stars (Maxted et al. 2001) and the focus of our study here. In the case that the He-flash occurs, zero-age horizontal branch models were artificialy created from a $2.25 M_{\odot}$ He-core-burning star, where we reset the chemical compositions to the values before the flash. This treatment is not rigorously valid. Full evolutionary models of the He-flash show that the $\mathrm{C}$ abundance in the He-core can increase up to $~ 5 \%$ (Piersanti et al. 2004; Serenelli \& Weiss 2005). Since the $p$-modes are not sensitive to the core, we are not worried about this. An interesting scenario is an $\mathrm{sdB}$ star that is formed by a late He-core flash on the white dwarf cooling curve (Castellani \& Castellani 1993). In such a case the He-flash-driven convection zone can penetrate into the H-rich layers, resulting in a surface enrichment of He and C (Brown et al. 2001; Schlattl et al. 2001; Cassisi et al. 2003). We note that this might influence the pulsations, but we will not discuss this scenario further here.

On the EHB, we used for temperatures $\log T>7$ the same opacities as mentioned above. In the outer layers of the star, $\log T<7$, where the pulsation driving region is located, the opacities were calculated by interpolating between several OPAL tables computed with iron abundance enhanced by factors of $f=1,2,5$, and 10 relative to solar, thus $X(\mathrm{Fe})=0.071794 Z f$. The abundances of the other heavy elements are decreased such that the overall metallicity is kept constant as in Miglio et al. (2007).

\subsection{The oscillation calculations and iron accumulation}

We adapted the Eggleton evolution code so that the output is suitable for pulsation calculations. In practice, this implied calculating some additional physical quantities during the evolution, and modifying the mesh to have sufficient meshpoints in the stellar envelope. The seismic properties of the stellar models are then calculated with two pulsation codes. The adiabatic code OSC by Scuflaire et al. (2007) is used to obtain the approximate frequencies, which are used as a first guess in the linear non-adiabatic code MAD by Dupret (2001). We determined the theoretical frequency spectrum up to $l=2$, since it is expected that higher order modes are geometrically cancelled. Charpinet et al. (1996) has established that the excitation of sdB oscillatons is related to a local enrichment of iron in the stellar envelope caused by diffusion. Radiative levitation is expected to set up significant chemical gradients within a diffusion timescale of $\sim 10^{5} \mathrm{yr}$, and consequently iron accumulates around $\log T \approx 5.3$ 
(Michaud et al. 1989; Chayer et al. 1995). Time-dependent diffusion calculations (Fontaine et al. 2006) show that, after $\sim 10^{5} \mathrm{yr}$, many pulsation modes are excited. Since element diffusion is not treated in the evolution code, we used an approximation for the iron accumulation, assuming that the iron only affects the stellar structure through the opacity. At each timestep of the evolution calculations, the iron enhancement factor $f$ is increased with a Gaussian centered at $\log T=5.3$,

$\frac{\mathrm{d} f}{\mathrm{~d} t}=\frac{(1-f / 10)^{3}}{\tau} \exp \left(-\frac{(\log T-5.3)^{2}}{\sigma^{2}}\right)$,

with the initial condition $f(t=0)=1$. The width $\sigma^{2}=0.05$ and accumulation timescale $\tau=4 \times 10^{5} \mathrm{yr}$ are chosen such that iron is only increased in the region $4.5<\log T<6.1$, and $\lim _{t \rightarrow \infty} f=10$, which is loosely based on the time-dependent diffusion calculations of Fontaine et al. (2006) and the equilibrium profiles of Charpinet et al. (1997). Our parametric approximation is rather ad hoc, but since we are interested in the relative differences between two different scenarios, the exact shape of the iron profile is not crucial here. We will discuss the influence of the iron profile on the pulsations and the evolution of the star in Sect. 3.1.

\section{Results}

\subsection{Effects of the iron accumulation}

In Fig. 1, we show $f$ throughout the star for different ages of an $\mathrm{sdB}$ star. Note that the temperature range $4.5<\log T<6.1$ corresponds to a very narrow mass shell of $\sim 10^{-6} M_{\odot}$. In Fig. 1c, we included the backreaction of convective mixing on our parametric iron profile during the evolution. Note that the iron abundance is homogeneous near $\log T=5.3(\log q=-10)$ and $\log T=4.6$ $(\log q=-12.5)$. This is caused by two narrow convective layers due to iron and helium ionization, respectively. Interestingly, we found that the convective region around $\log T=5.3$ would not be present without iron accumulation. We determined that the slightly perturbed iron profile has a negligible effect on the driving and the pulsation frequencies. Moreover, since our description of iron accumulation is approximate, we have not included this effect in subsequent calculations.

In Fig. 2, we show the effect of different iron abundance profiles on the $\mathrm{sdB}$ evolution in the $\log g-T_{\text {eff }}$ diagram. We compared our parametric approach (Eq. (3)) with the case of no iron enhancement, and a uniform enhancement of $f=10$ in the whole envelope, as used in studies of mode excitation (Jeffery \& Saio 2006). It is evident that using $f=10$ influences the evolution drastically, while our Gaussian parametrization of $f$ has little effect. Higher iron abundances indeed give higher opacities, and thus larger stellar radii. With Eq. (3) we only increase iron in a relatively small region, resulting in a minimal effect on the stellar structure. However, a minimal change of the stellar structure can result in a visible shift of the pulsation frequencies, as shown by Fontaine et al. (2006).

We also compared the effect on the excitation, and find that our approximation of the iron profile can excite almost as many modes as the $f=10$ enhancement, see Figs. 3a,c. This is understood in terms of the driving mechanism in sdB stars, which is associated with the iron opacity bump such that accumulating iron in this driving region is sufficient for the excitation of the pulsation modes, see Figs. 3d,f. Thus, with our parametric approach to iron accumulation, the issue of excitation can be addressed while keeping the effect of the iron profile on the stellar
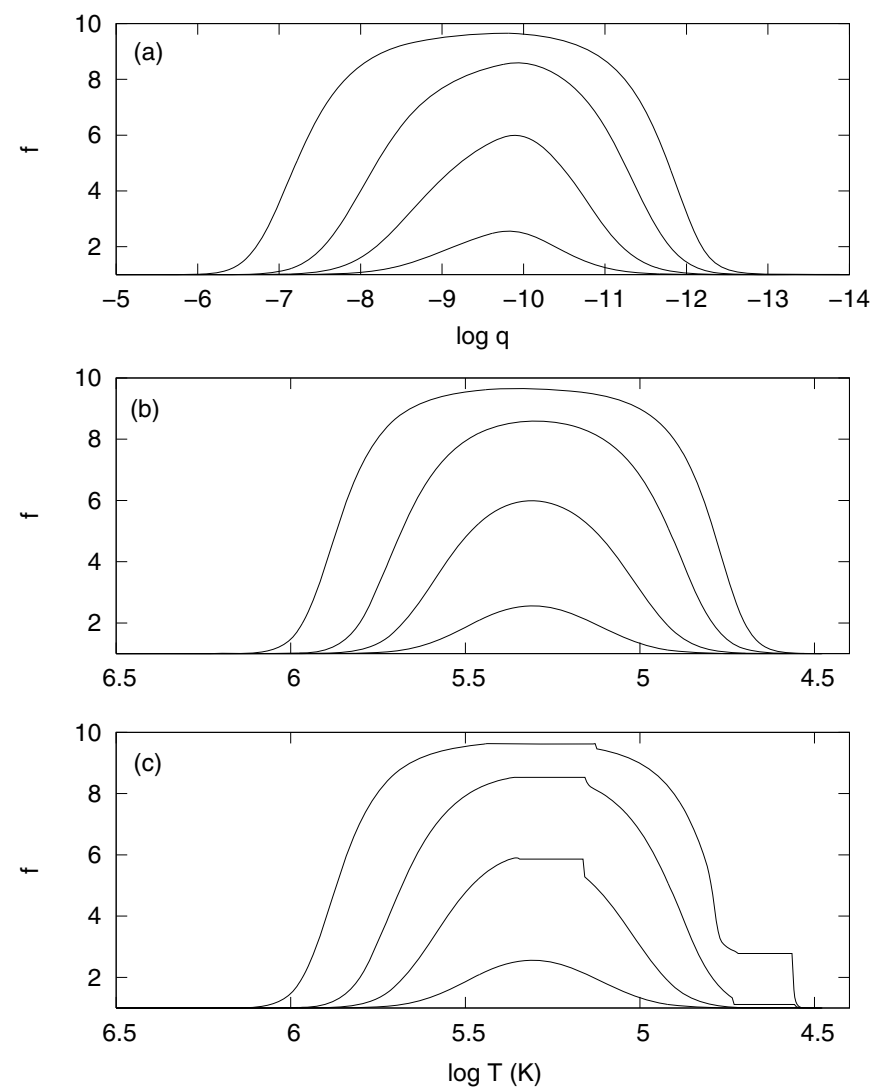

Fig. 1. The iron enhancement factor $f$ throughout the star as a function of a) the outer mass fraction: $\log q=\log \left(1-\frac{M_{r}}{M_{*}}\right)$, and b) the temperature. In panel $\mathbf{c}$ ), we show the effect of convective mixing on our parametric iron profile. The sdB star has $M_{\text {core }}=0.47 M_{\odot}$ and $M_{\text {env }, 0}=10^{-4} M_{\odot}$, and was constructed from a $1.00 M_{\odot}$ ZAMS model. The profiles from bottom to top correspond to sdB ages $10^{5}, 10^{6}, 10^{7}$, $1.8 \times 10^{8} \mathrm{yr}$, where the last model is at the end of core-He-burning.

structure realistic. Furthermore, these are the first evolutionary models of sdB stars that include the effect of iron accumulation, albeit in an approximative manner.

\subsection{The stellar models}

Following the procedure as described in Sect. 2, we constructed a grid of canonical, i.e. post-He-flash, sdB models (hereafter called grid $A$ ) with masses in the range $0.42-0.47 M_{\odot}$ in steps of $0.01 M_{\odot}$. The maximum mass we obtained for the degenerate core of an RGB star is $0.47 M_{\odot}$, thus we did not consider postflash sdB stars above this mass. The H-envelope masses, $M_{\mathrm{env}, 0}$, considered are $0.0001,0.0003$, and $0.0006 M_{\odot}$, where $M_{\mathrm{env}, 0}$ is defined as the total mass of the hydrogen content directly after the removal of the envelope. Thus, we have $18 \mathrm{sdB}$ evolution tracks, which we followed until the end of He-core burning. After each $10^{7} \mathrm{yr}$ of sdB evolution, the seismic properties were calculated, and we only considered models with unstable modes. We have not found unstable modes in our post-He-core burning models. Therefore our analysis is limited to sdB stars in their He-core burning phase, leading to a total of 402 seismic models in the range of $T_{\text {eff }}=25000-34000 \mathrm{~K}$ and $\log g=5.4-6.0$.

The grid of non-canonical sdB stars (hereafter called grid $B$ ) consists of 5 tracks: $\left(M_{\mathrm{sdB}}\left(M_{\odot}\right), M_{\mathrm{env}, 0}\left(M_{\odot}\right)\right)=(0.44,0.005)$, $(0.45,0.005),(0.46,0.005),(0.47,0.0075)$, and $(0.47,0.005)$. 


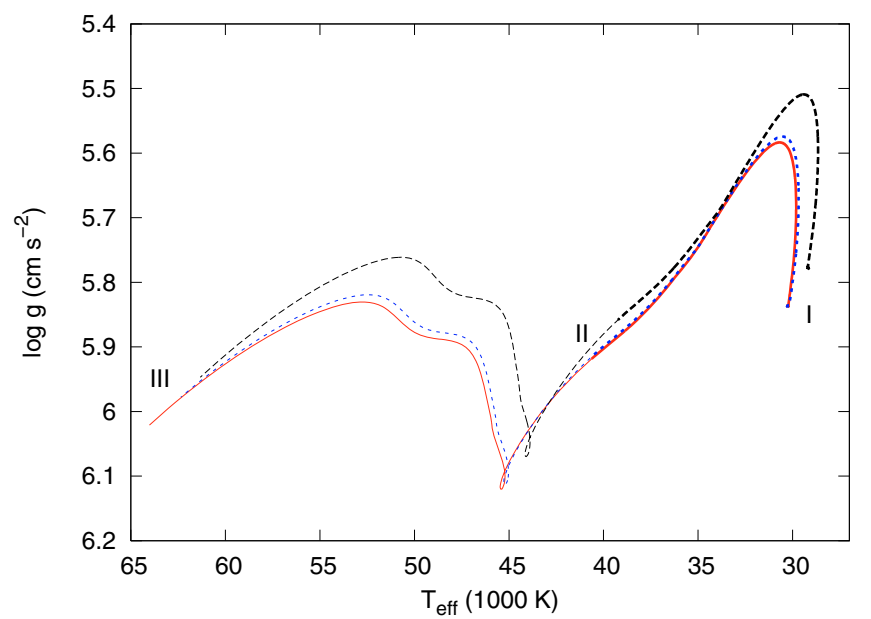

Fig. 2. Evolutionary tracks in the $\log g-T_{\text {eff }}$ diagram of sdB stars with different iron profiles. The three tracks start with the same zero-age EHB model with $M_{\mathrm{sdB}}=0.47 M_{\odot}$ and $M_{\mathrm{env}, 0}=10^{-4} M_{\odot}$, created from a $1.00 M_{\odot}$ ZAMS model. The lowest solid curve is for a model with no iron enhancement, the middle dotted curve is for a Gaussian iron increase centered at $\log T=5.3$, and the upper dashed curve is for iron increased with a factor 10 uniformly in the envelope. The labels I, II, II indicate the ZAHB, the end of core-He burning and the end of He-shell burning, respectively. It takes the $\mathrm{sdB}$ star $1.8 \times 10^{8} \mathrm{yr}$ to evolve from I to II, and $10^{7} \mathrm{yr}$ from II to III.

Along these tracks we have in total 98 seismic models, again taken after each $10^{7} \mathrm{yr}$ of sdB evolution. In Table 1 , more details about the models are given.

The sdB evolution tracks and the seismic models can be seen in Fig. 4. The tracks start directly after the removal of the envelope. For the post-flash models, this corresponds to the zero-age EHB. The post-non-degenerate models have hydrogen extending to deeper layers, hence allowing some H-shell-burning, before reaching the zero-age EHB. Note that, although the two different types of sdB stars can evolve through the same $\log g-T_{\text {eff }}$ during core-He-burning, the post-He-core-burning evolution differs, see Fig. 4b. The post-non-degenerate sdB star again has a short phase $\left(\sim 10^{6} \mathrm{yr}\right)$ of $\mathrm{H}$-shell burning, before starting He-shell burning.

\subsection{Comparing two representative models}

We examined the physical differences in the interior structure of a post-flash $(\alpha)$ and a post-non-degenerate $(\beta)$ sdB with same

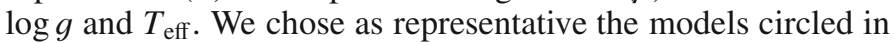
Fig. $4 \mathrm{~b}$ at $\log g=5.78$ and $T_{\text {eff }}=30 \mathrm{kK}$. One of the main differences is the abundance profiles, see Fig. 6a. The He-H transition layer of $\beta$ is much broader and located deeper in the star. The envelope of $\beta$ is in the region where the shrinking convective core passed through during the MS, hence the low $\mathrm{H}$-abundance here: $X=0.18$. For $\alpha$, the helium core has grown into the region that used to be part of the convective envelope during the RGB, and, as a result, the $\mathrm{He}-\mathrm{H}$ transition region is much narrower while the $\mathrm{H}$-abundance in the envelope is around $X=0.66$. We will discuss the possibility of diffusion of hydrogen to the surface in Sect. 4. In Fig. 6d, we show the iron mass fractions, and in Fig. 6e the resulting opacity profiles. The outer opacity bump near $\log T=4.7$ is associated with helium ionization, and the iron opacity bump near $\log T=5.3$ is enhanced by the local iron accumulation. (a)

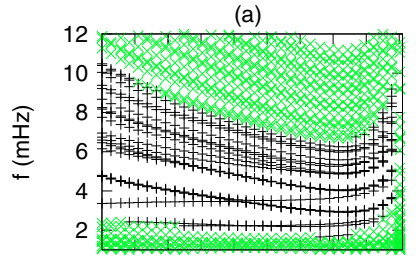

(b)

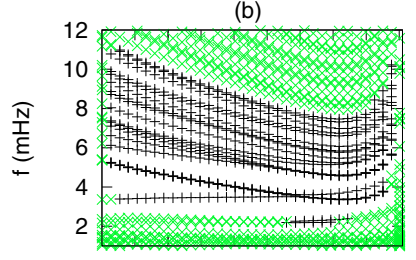

(c)

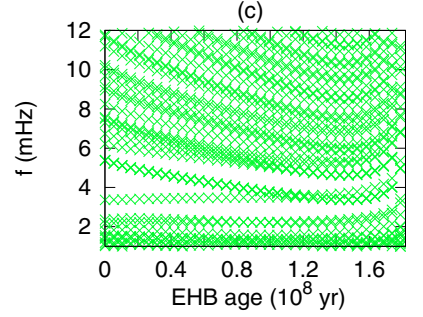

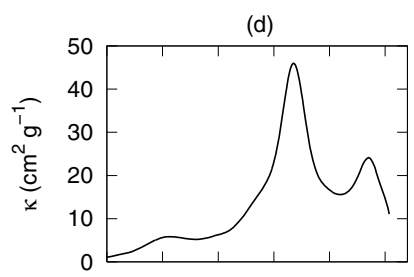

(e)

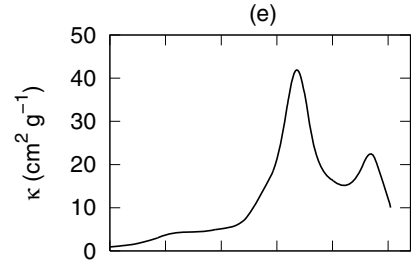

(f)

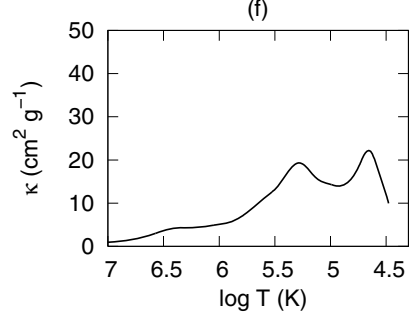

Fig. 3. Left: the frequencies of the stable $(\times)$ and unstable $(+)$ modes $(l \leq 2)$ as a function of the EHB age, for the three tracks given in Fig. 2. Right: the opacity as a function of the temperature after $10^{7} \mathrm{yr}$ of $\mathrm{sdB}$ evolution. Panels $\mathbf{a}), \mathbf{d})$ correspond to $f=10, \mathbf{b})$, e) are for the Gaussian parametrization of $f$, and $\mathbf{c}), \mathbf{f}$ ) are for the model with no iron enhancement.

Two important quantities in stellar pulsation theory are the the Brunt-Väisäla frequency $N$ and the Lamb frequencies $L_{l}$,

$N^{2}=\frac{G m}{r^{2}} \frac{\delta}{H_{\mathrm{p}}}\left(\nabla_{\mathrm{ad}}-\nabla+\frac{\varphi}{\delta} \nabla_{\mu}\right)$

with $\quad \delta=-\frac{\partial \ln \rho}{\partial \ln T} \quad$ and $\quad \varphi=\frac{\partial \ln \rho}{\partial \ln \mu}$

$L_{l}^{2}=\frac{l(l+1) c_{\mathrm{s}}^{2}}{r^{2}}$

where $\mu$ is the molecular weight and $c_{\mathrm{s}}$ is the adiabatic sound speed. When $N^{2}<0$, the Ledoux criterion for dynamical stability is violated (Ledoux 1947). Thus, in Figs. 6b,c, the convective regions can be clearly identified. The innermost one is related to the convective core, and the outer two are the narrow convective layers due to iron (near $\log T=5.3$ ) and helium ionization (near $\log T=4.6$ ). Also, chemical gradients are apparent in $N^{2}$ in the form of localized peaks. The peak near the center is identified with the $\mathrm{C}-\mathrm{O} / \mathrm{He}$ transition zone and the outermost peak to the $\mathrm{He}-\mathrm{H}$ transition zone. The Lamb frequencies are plotted in the same figure to indicate the propagation zones of the $g$-modes, $\sigma^{2}<\left(N^{2}, L_{l}^{2}\right)$, and the $p$-modes, $\sigma^{2}>\left(N^{2}, L_{l}^{2}\right)$, where $\sigma$ is the angular pulsation frequency. It is apparent that $g$-modes are deep interior modes, while $p$-modes probe the superficial outer layers as pointed out by Charpinet et al. (2000).

Clearly, models $\alpha$ and $\beta$ have very different physical characteristics. To establish how this affects their seismic properties, we compare their frequencies in Fig. 5. Since the large frequency separation $\Delta f=f_{n, l}-f_{n-1, l}$ is mainly dependent on the dynamical timescale, we see that $\Delta f$ at high frequencies is more or less the same for the two models. The lower 

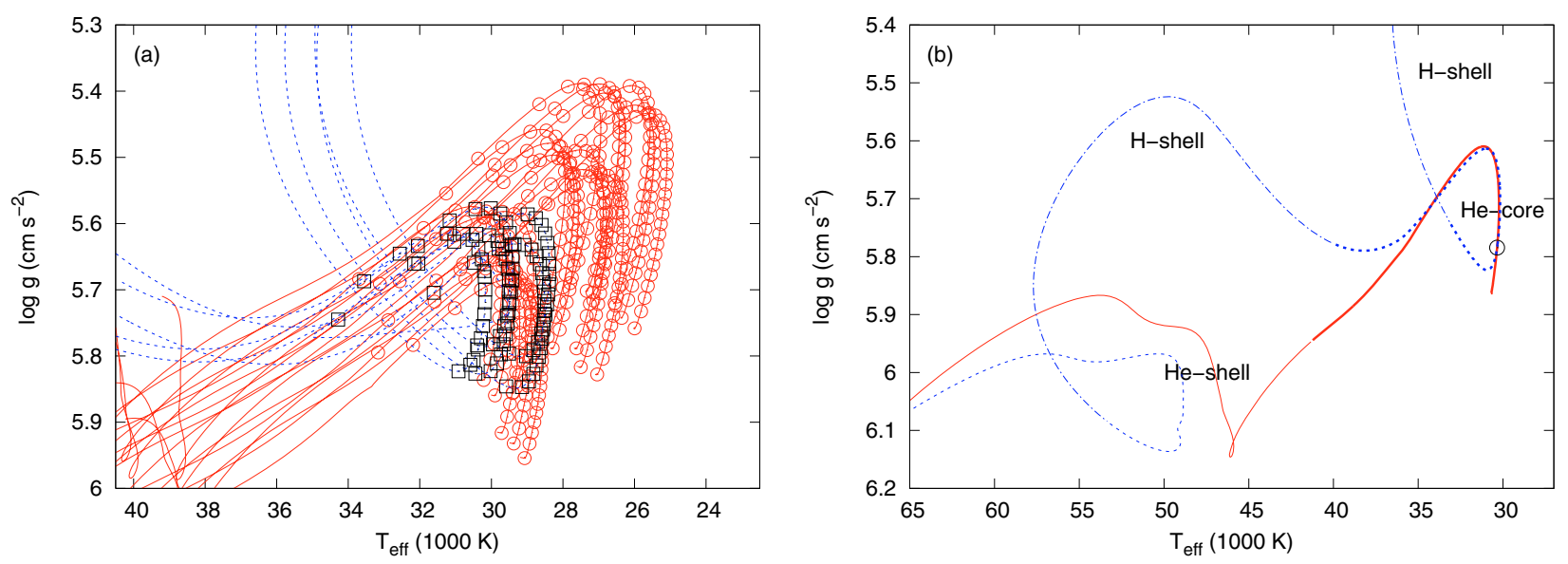

Fig. 4. a) sdB Evolutionary tracks in the $\log g-T_{\text {eff }}$ diagram. The solid curves correspond to the post-flash sdB models, and the dotted curves to the post-non-degenerate sdB models. The circles and squares indicate models on which we performed pulsation calculations for the post-flash and post-non-degenerate tracks respectively. b) Evolutionary tracks of a post-flash sdB star created from of low-mass $\left(M_{\text {ZAMS }}=1.00 M_{\odot}\right)$, and a post-non-degenerate sdB star created from a high mass $\left(M_{\text {ZAMS }}=3.00 M_{\odot}\right)$ progenitor, given by the solid and dotted curve respectively. Along the tracks the dominant energy source (either H-shell-burning, He-core-burning or He-shell-burning) is noted. The bold part of the tracks indicate the He-core-burning phase and the thin part the He-shell burning phase. During the dot-dashed parts of the post-non-degenerate track, H-shell burning is the dominant energy source. At the circle in $\mathbf{b}$ ), we selected from both tracks a model for a detailed comparison, see Sect. 3.3.

Table 1. The models in grid $A$ and $B^{a}$.

\begin{tabular}{lllll}
\hline \hline Track & \# models & $M_{\text {sdB }}\left(M_{\odot}\right)$ & $M_{\text {env }, 0}\left(M_{\odot}\right)$ & $M_{\text {ZAMS }}\left(M_{\odot}\right)$ \\
\hline$A 1$ & 27 & 0.42 & 0.0001 & 1.85 \\
$A 2$ & 27 & 0.42 & 0.0003 & 1.85 \\
$A 3$ & 27 & 0.42 & 0.0006 & 1.85 \\
$A 4$ & 25 & 0.43 & 0.0001 & 1.80 \\
$A 5$ & 25 & 0.43 & 0.0003 & 1.80 \\
$A 6$ & 25 & 0.43 & 0.0006 & 1.80 \\
$A 7$ & 23 & 0.44 & 0.0001 & 1.75 \\
$A 8$ & 23 & 0.44 & 0.0003 & 1.75 \\
$A 9$ & 23 & 0.44 & 0.0006 & 1.75 \\
$A 10$ & 21 & 0.45 & 0.0001 & 1.65 \\
$A 11$ & 21 & 0.45 & 0.0003 & 1.65 \\
$A 12$ & 21 & 0.45 & 0.0006 & 1.65 \\
$A 13$ & 20 & 0.46 & 0.0001 & 1.55 \\
$A 14$ & 20 & 0.46 & 0.0003 & 1.55 \\
$A 15$ & 20 & 0.46 & 0.0006 & 1.55 \\
$A 16$ & 18 & 0.47 & 0.0001 & 1.00 \\
$A 17$ & 18 & 0.47 & 0.0003 & 1.00 \\
$A 18$ & 18 & 0.47 & 0.0006 & 1.00 \\
$B 1$ & 23 & 0.44 & 0.005 & 2.75 \\
$B 2$ & 18 & 0.45 & 0.0075 & 2.75 \\
$B 3$ & 21 & 0.46 & 0.005 & 2.90 \\
$B 4$ & 18 & 0.47 & 0.0075 & 2.90 \\
$B 5$ & 18 & 0.47 & 0.005 & 3.00 \\
\hline
\end{tabular}

${ }^{a}$ The label of the track given in the first column is used for reference in Fig. 7. The second column gives the number of seismic models along that track. The final column shows the mass of the ZAMS models from which the sdB star is created.

frequencies, however, are in fact mixed modes that are more sensitive to the core, and we see a better distinction between models $\alpha$ and $\beta$ here. Moreover, the frequency ranges of excited $l=0-2$ modes are not the same for these two models; for model $\alpha$ this range is [3.4 $\mathrm{mHz}, 10.6 \mathrm{mHz}$, and for model $\beta$, it is $[5.7 \mathrm{mHz}, 14.2 \mathrm{mHz}]$. The excited modes thus have lower frequencies in model $\alpha$ than in model $\beta$. To understand this, we compare in Fig. 6h the work integral of these two models for the radial mode $p_{7}$. The work integral increases towards the surface in the driving region and decreases towards the surface in damping regions. The surface value is the dimensionless growth rate, positive for unstable modes and negative for stable ones. We see that $p_{7}$ is unstable in model $\beta$, but stable in model $\alpha$, which can also be seen in Fig. 5.

A first possible origin of the differences could come from the opacity, since the driving is a $\kappa$-mechanism operating in the iron opacity bump. Figure 6e shows that the opacity is slightly larger for model $\beta$. The driving is thus a little more efficient in model $\beta$. But this is not the main source of differences. Since the envelope $\mathrm{H}$-fraction is much smaller in model $\beta(X=0.18)$ than in model $\alpha(X=0.66)$, the molecular weight is larger and, at given temperature, the density is significantly higher $(\sim 1.5 \times)$ in model $\beta$, as shown by Fig. 6f. The driving of the modes is related to the opacity, which is mainly a function of temperature. Hence, if the eigenfunctions of two given modes have the same shape as a function of temperature, the driving is the same. Here we compare the modes $p_{7}$ of two models with the same radius. As it is usually found for $p$-modes, their last node is located at the same geometrical distance from the surface $\Delta r$. But the gradient of temperature is not the same for the two models: $|\mathrm{d} T / \mathrm{d} r| \propto \kappa \rho$ is greater in model $\beta$ because of the higher density, as can be seen in Figs. 6f,g. Hence, the difference between the temperature at the last node and at the surface, $|\Delta T| \simeq|\mathrm{d} T / \mathrm{d} r| \Delta r$, is greater in model $\beta$ than in model $\alpha$. This is exactly what we find in Fig. 6i, where the eigenfunction $|\delta T / T|$ is given. In terms of the temperature, the last node is closer to the surface in model $\alpha$ than it is in model $\beta$. To get the same driving as in model $\beta$, the last node of model $\alpha$ would have to be deeper in the star, which is only possible by considering a mode with lower radial order and frequency. Hence, the frequencies of excited modes are lower in model $\alpha$ than in model $\beta$.

\subsection{Comparing two grids of models}

We investigated if it is possible to distinguish between a post-Heflash $(a)$ and a post-non-degenerate sdB stellar model $(b)$ from 


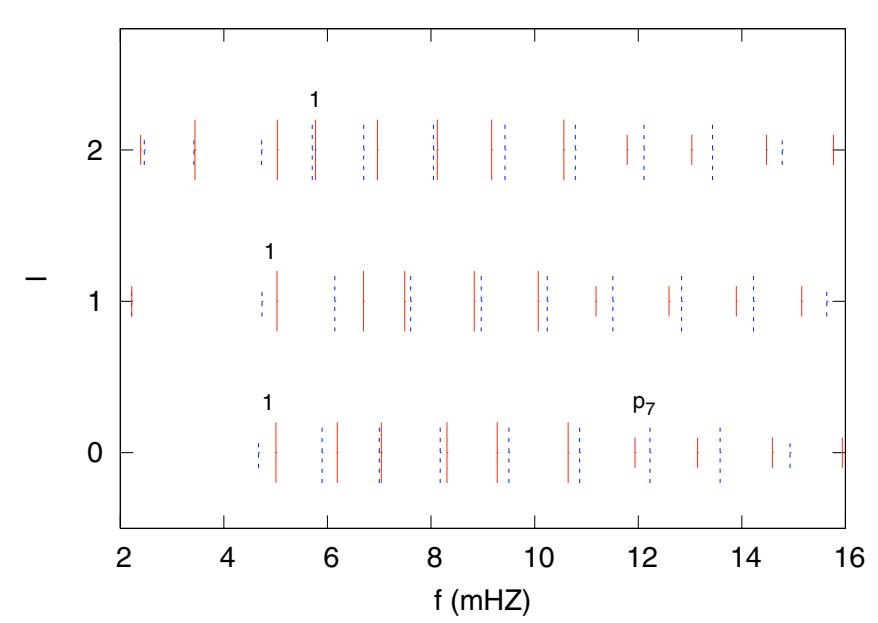

Fig. 5. The pulsation frequencies for modes $l=0-2$. Solid line segments are for model $\alpha$ and dotted ones for model $\beta$. Unstable modes are given by long line segments, and stable modes by short line segments. The modes with radial order $n=1$ and radial mode $p_{7}$ are indicated.

observed oscillation modes. Imagine we observed the frequencies of $b$; is it then possible to find an acceptable seismic match in our grid of canonical post-flash models (grid $A$ )? We took as "observed" frequencies those of unstable modes up to $l=2$. We did this for each model $b$ in grid $B$, thus finding the best seismic match within grids $A$ and $B$.

Since frequency separations follow from asymptotic relations for $p$-modes, the frequency is a natural quantity for model comparison. Despite this, periods have been used more often in the literature so far, when comparing observed modes of sdB stars with those predicted by models. We also considered period matching, but found frequency matching more suitable to compare the $p$-modes of the models. This will be different for $g$-modes, where the mode period is the natural quantity to comparing observations with models.

To quantify "acceptable", we used the merit function

$\mathcal{M}^{2}=\frac{1}{n_{b}} \sum_{i=1}^{n_{b}}\left(f_{a}^{i}-f_{b}^{i}\right)^{2}$,

where $f_{b}^{i}$ is one of the $n_{b}$ excited frequencies of star $b$, and $f_{a}^{i}$ is the correspondingly matched frequency of star $a$, expressed in $\mathrm{mHz}$. The frequency matching is done such that $\mathcal{M}^{2}$ is minimized by brute-force fitting. It is clear that the lower $\mathcal{M}^{2}$, the better the match between $a$ and $b$. Matches with $\mathcal{M}^{2}>0.05$ are considered unacceptable, which is a generous limit, as we will see later. We investigated four different scenarios:

(i) we are not able to identify the modes, $\log g$ and $T_{\text {eff }}$ of the "observed" star $b$ are unknown, and the "observed" frequencies are allowed to be matched with both stable and unstable frequencies of the "theoretical" model $a$;

(ii) same as (i), except the modes are identified, thus the $l$-value must be matched;

(iii) same as (i), except $\log g$ and $T_{\text {eff }}$ are known within errors of $\mathrm{d} \log g=0.1$ and $\mathrm{d} T_{\text {eff }}=1000 \mathrm{~K}$;

(iv) same as (i), except the "observed" frequencies are only matched with unstable "theoretical" frequencies, i.e. assuming that the theory correctly predicts which frequencies are exited and which are not.

In Fig. 7, we show $\mathcal{M}^{2}$ for each gridpoint in grids $A$ and $B$ for the scenarios (i)-(iv). The matches with low $\mathcal{M}^{2}$ are visible as dark diagonal regions, This is a result of the change in frequencies during the sdB evolution. From Fig. 7(i)-(iii), it is clear that the distinction between models $a$ and $b$ is drastically increased if we have either mode identification or spectroscopic $\log g$ and $T_{\text {eff }}$ values. Figure 7(iv) shows that, if we only allow matching to unstable (and not stable) frequencies of $a$, there are no matches.

The matches with lowest $\mathcal{M}^{2}$ are circled in Fig. 7 and details of these models are shown in Fig. 8 and Table 2. For all scenarios the same model $b$ gives the best match, namely the last model of evolutionary track $B 5$. We understand that in terms of only the higher frequencies with radial order $n \geq 3$ being excited in this model. As we discussed in Sect. 3.3, the lower frequencies are more sensitive to the deeper layers, thus the distinction between models $a$ and $b$ is better detected at low frequencies.

As a comparison to a real case, we considered the optimal model for PG 0014+067, for which Brassard et al. (2001) found $\chi^{2}=0.5374$, where $\chi^{2}$ is a merit function based on mode period comparison. Translated to our frequency merit function, this is equivalent to $\mathcal{M}^{2}=0.0084$. Although we find, in principle, seismic matches between $a$ and $b$ with $\mathcal{M}^{2}$ of this order for scenarios (i)-(iii), they are not statistically favoured. For scenario (i) we find that 12 of the 98 models in grid $B$ can be matched with a model in grid $A$ with $\mathcal{M}^{2} \leq 0.01$, and this is 7 for scenario (ii), and only 1 for scenario (iii).

\section{Discussion and conclusions}

We studied the so far neglected, post-non-degenerate sdB stars and compared their physical and seismic characteristics with those of canonical post-flash sdB stars, both formed in the $\mathrm{CEE}$ channel. The results presented here are a first step in distinguishing these two kinds of sdB stars on the basis of their observed oscillation character, which is necessary if seismic modelling is to achieve reliable mass determination. Furthermore, the observation of a post-non-degenerate sdB star in a post-CE binary would give strong constraints on the CE evolution. We plan to continue such investigations with an application to the $\mathrm{sdB}$ pulsator in the post-CE, eclipsing binary PG 1336-018 which we started in Hu et al. (2007) and Vučković et al. (2007).

We find that, in principle, a post-non-degenerate sdB star may appear as an EC 14026 star with similar pulsation frequencies as the canonical post-He-flash sdB star, although it is not likely. Additional observables, such as spectroscopic $\log g$ and $T_{\text {eff }}$ determinations and/or empirical mode identification from observables enable us to distinguish the two types of sdB stars more decisively. The frequency range of the unstable modes is also an important discriminator between the two formation channels. In general, for the same $\log g$ and $T_{\text {eff }}$ values, the excited frequencies of the post-non-degenerate sdB star are higher than the excited frequencies of the post-flash star. This is a direct result of the differing interior structures. Thus, special attention must be paid when observed frequencies are matched with theoretically predicted ones of modes that are not excited.

Up to now, there have not been any evolutionary models of sdB stars available that include the coupling between diffusion and evolution consistently. This is a deficiency, since iron accumulation due to radiative levitation is responsible for the pulsational instability in these stars (Charpinet et al. 1996). Also, it has been shown by Fontaine et al. (2006) that the iron accumulation changes the frequencies significantly. In our study, we have parametrized the iron accumulation, so that we can, at least in an approximative manner, simultaneously take into account the effects of iron enhancement and evolution on the pulsation modes. 

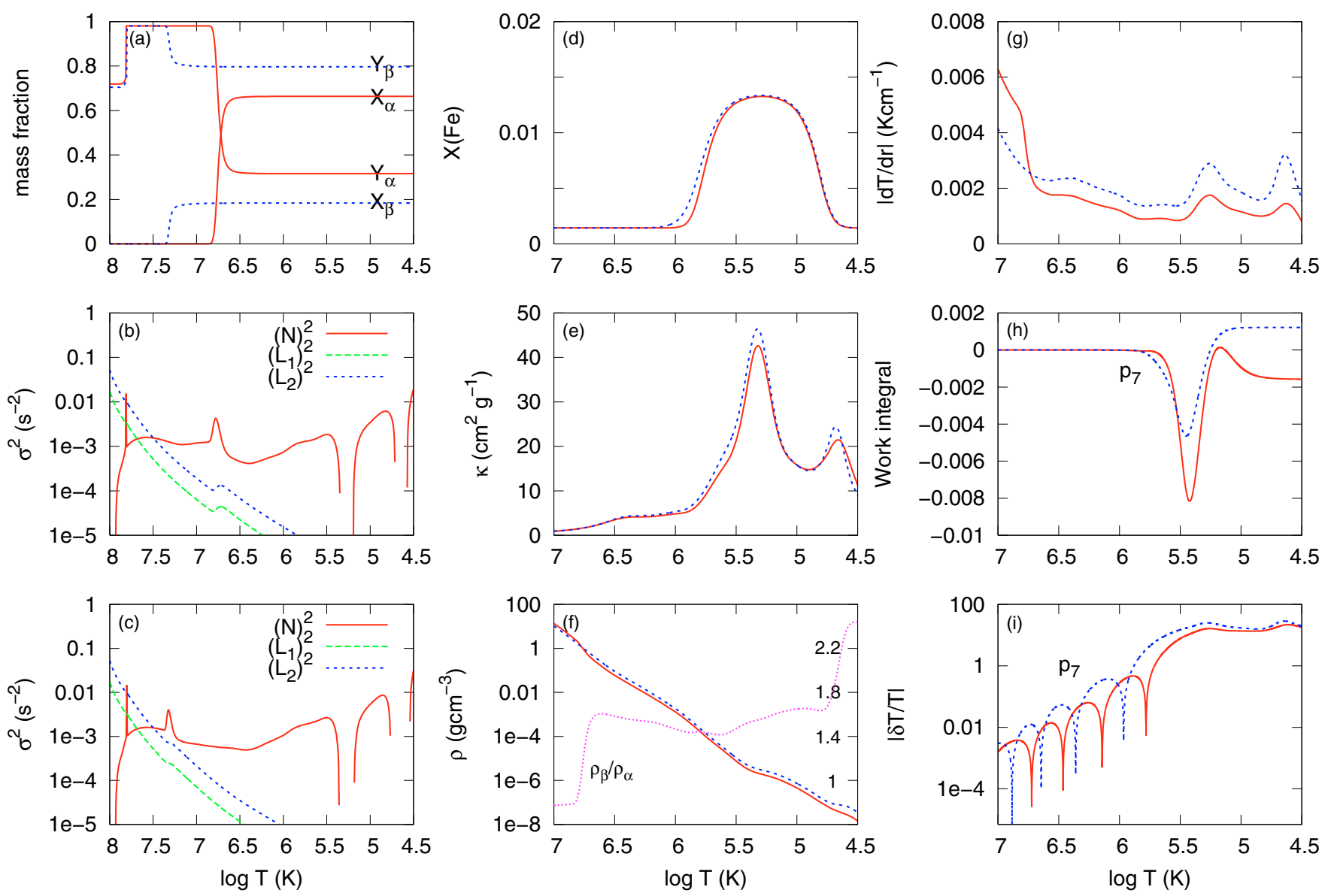

Fig. 6. Physical and seismic quantities of the two representative models $\alpha$ (post-flash) and $\beta$ (post-non-degenerate). The profiles are shown as a function of the temperature: a) the hydrogen $(X)$ and helium $(Y)$ mass fractions; b), c) the Brunt-Väisäla and Lamb frequencies for model $\alpha$ and $\beta$, respectively; d) the iron mass fraction; e) the opacity; f) the density, we also plotted the density ratio $\rho_{\beta} / \rho_{\alpha}$ on the right axis; g) the temperature gradient $|\mathrm{d} T / \mathrm{d} r|$; h) the work integral for radial mode $p_{7}$; and i) the eigenfunction $|\delta T / T|$ for the radial mode $p_{7}$. In all panels except b), c), solid lines represent model $\alpha$ and dotted lines model $\beta$. Note that panels a)-c) give the profiles throughout the entire star, while the profiles in panels d)-h) are for the stellar envelope.

Here we have not considered the influence of the other diffusive processes, i.e. diffusion due to gradients of pressure, temperature, and concentration. To a certain extent this can affect our results, because one of the main differences between the two types of sdB stars is the chemical composition of the stellar envelope. Specifically, we find in the envelope of the postnon-degenerate sdB star an $\mathrm{H}$-mass fraction of $X=0.18$, while the post-flash sdB star has $X=0.66$ there. Normally, it is assumed that sdB stars have $\mathrm{H}$-rich or even pure $\mathrm{H}$-envelopes, caused by gravitational settling. While this is true for the outermost layers, diffusion is not expected to work efficiently at depths $\log q \gtrsim-3$ (Richard et al. 2002; Michaud et al. 2007). Since the envelopes of the post-non-degenerate sdB stars extend to $\log q \gtrsim-2$ (i.e. $T \gtrsim 10^{7} \mathrm{~K}$ ), we do not expect diffusion to wash away all the qualitative differences in the chemical profiles, although the differences may be less pronounced. Diffusion, however, will significantly change the surface abundances of our models and likely will bring them into agreement with the observed values. Spectroscopic line profile analysis has shown that the majority of sdB is He-deficient, and only a few are He-rich (Edelmann et al. 2003; Lisker et al. 2004). Stellar evolution models that include diffusion coupled to reliable atmosphere models are needed to assess whether the two different formation channels will be distinguishable via a spectroscopic abundance analysis. We are currently computing such evolutionary sdB models including diffusion due to gradients of pressure, temperature, and concentration. Our preliminary results indeed agree with our expectations, i.e. the $\mathrm{H}$-surface abundance increases on a very short timescale, but the chemical profiles at deeper layers are not affected. The pulsational properties of these improved models will be discussed in detail in a forthcoming paper.

We have made a modest grid of models that is sufficient for our comparative study. Detailed seismic modelling of an observed star, however, will require a finer grid. For now, we have chosen not to make sdB models above $0.47 M_{\odot}$, since this is the maximum mass the degenerate $\mathrm{He}$-core of a red giant with $Z=0.02$ can have before experiencing the He-flash. A metallicity of $Z=0.004$ allows the He-core to grow up to $0.48 M_{\odot}$ on the RGB. However, we find that, in order to excite modes in these low metallicity stars, an iron enhancement greater than a factor 10 is required. This was to be expected, since Charpinet et al. (1996) found unstable pulsation modes for models with uniform $Z \geq 0.04$ in the H-rich envelope. We have therefore not pursued these models further. The question whether post-flash sdB stars can have masses $>0.47 M_{\odot}$ is also closely related to the input physics (e.g. convective overshooting) and the physics of the He-flash, and needs to be examined further. 
(i)

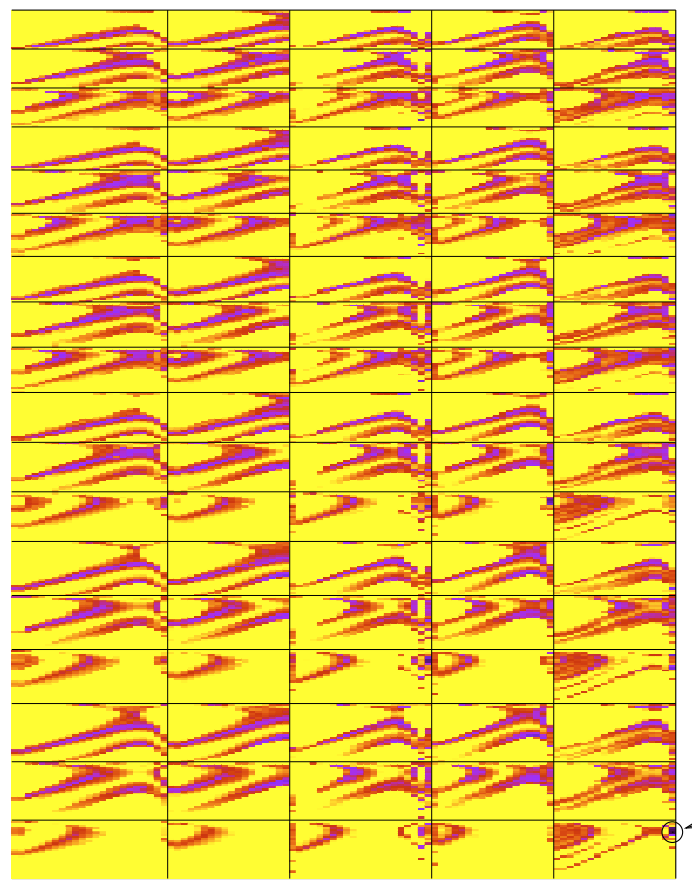

(iii)

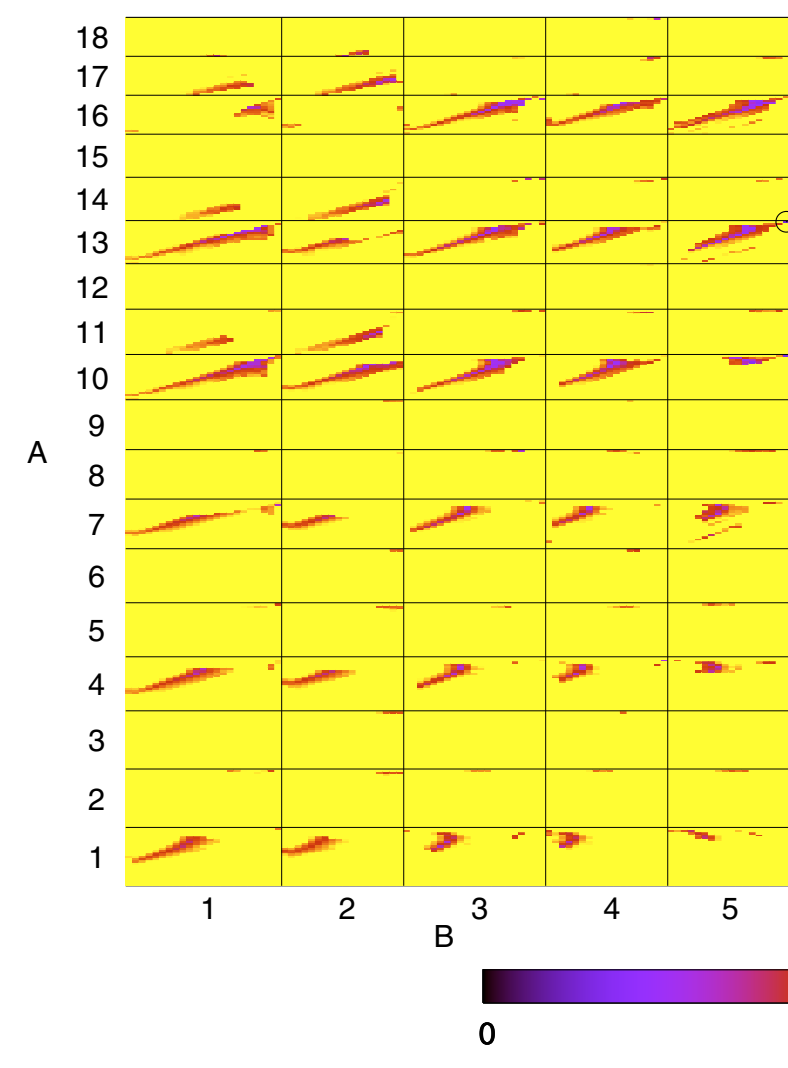

(ii)

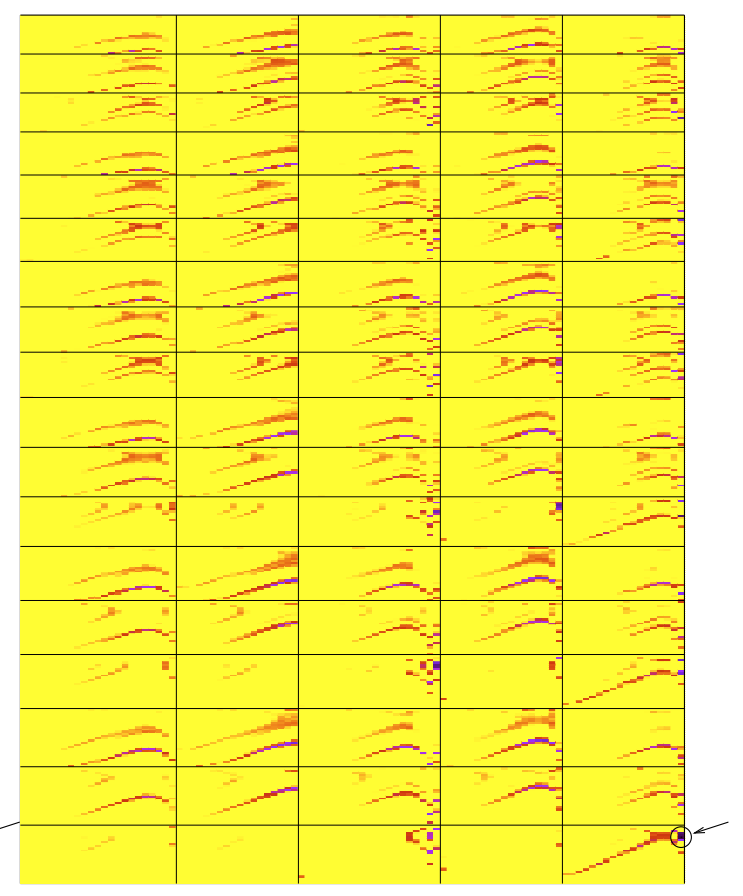

(iv)

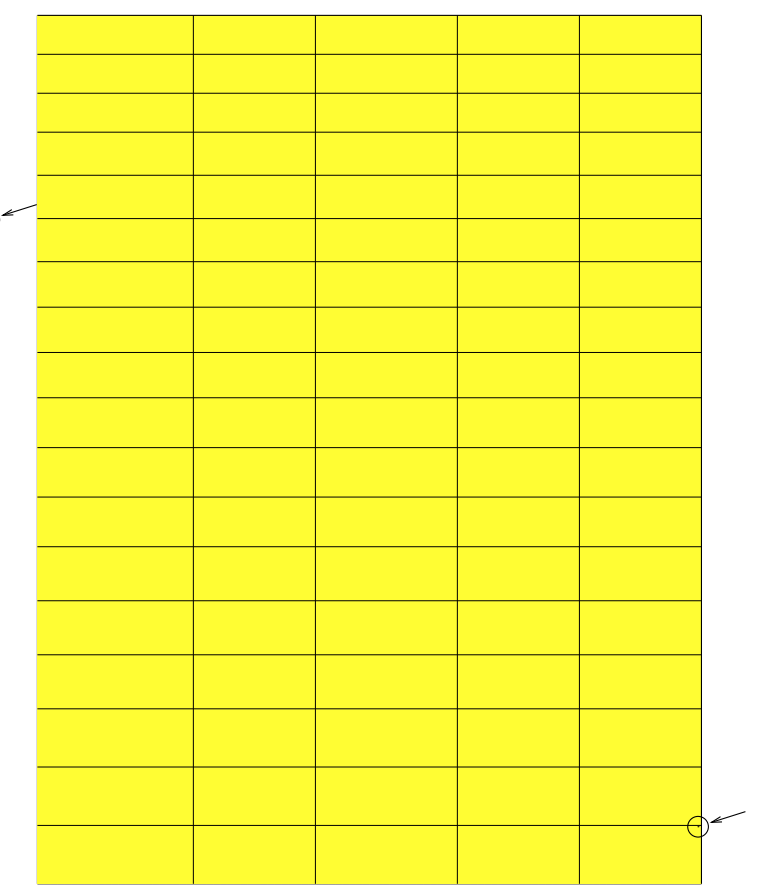

Fig. 7. Colourmap of $\mathcal{M}^{2}$. The models of grid $A$ are plotted along the vertical axis, and the models of grid $B$ along the horizontal axis. The models are divided in blocks according to evolutionary track, where we have ordered the blocks with increasing mass (see Table 2), and in the blocks the models are ordered with increasing age. The four panels are for the four different scenarios i), ii), ii), and iv), as described in the text. In each panel we have pointed out and circled the gridpoint with minimum $\mathcal{M}^{2}$. The frequency matching of these gridpoints can be seen in Fig. 8. Note that panel iv) has no acceptable matches, but we still circled the one with the lowest $\mathcal{M}^{2}$. 

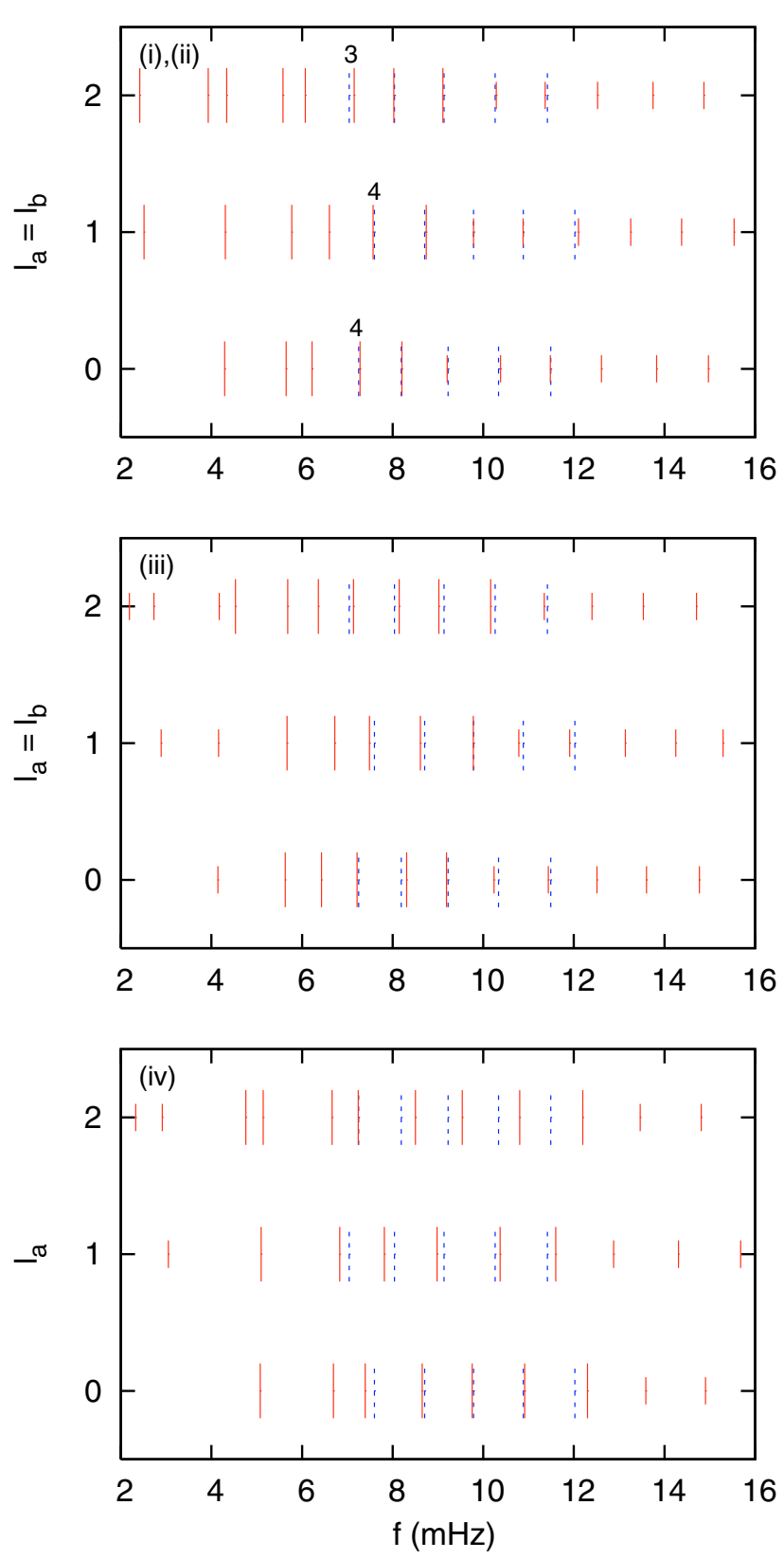

Fig. 8. The frequency matches of the minimum $\mathcal{M}^{2}$ gridpoints. The $l$-value of model $a$ is depicted on the vertical axis. For scenarios i) and ii), this is equal to the $l$-value of model $b$, for scenario iv) it is not. Solid line segments are for model $a$ and dotted ones for model $b$. In the upper panel, we have indicated the lowest radial order of the unstable modes of model $b$.

In this paper, we have focused on the short-period $p$-mode $\mathrm{sdB}$ pulsators. The case of the long-period $g$-mode $\mathrm{sdB}$ pulsators is, although challenging from an observational point of view, an additional very interesting theoretical case study. The $p$-modes only probe the outermost layers, and hence are less affected by the differing composition gradients than the $g$-modes, as they propagate deeper into the star. The long-period sdB pulsators are interpreted as cooler sdB models with much thicker hydrogenenvelopes than the short-period sdB pulsators (Fontaine et al. 2003; Jeffery \& Saio 2006). Since the $g$-modes are deep interior modes, full evolutionary models including iron accumulation, as developed here, are required to model these stars. At present,
Table 2. The models with minimum $\mathcal{M}^{2}$ for the four different scenarios.

\begin{tabular}{llll}
\hline \hline & (i) \& (ii) & (iii) & (iv) \\
$\mathcal{M}^{2}$ & 0.0018 & 0.0082 & 0.080 \\
\hline Track & $A 1$ & $A 13$ & $A 1$ \\
$M_{\text {sdB }}\left(M_{\odot}\right)$ & 0.42 & 0.46 & 0.42 \\
$M_{\text {env }, 0}\left(M_{\odot}\right)$ & 0.0001 & 0.0001 & 0.0001 \\
$\log g$ & 5.69 & 5.69 & 5.78 \\
$T_{\text {eff }}(\mathrm{K})$ & 29216 & 33117 & 32187 \\
EHB age $(\mathrm{yr})$ & $2.2 \times 10^{8}$ & $1.9 \times 10^{8}$ & $2.7 \times 10^{8}$ \\
\hline Track & $B 5$ & $B 5$ & $B 5$ \\
$M_{\text {sd }}\left(M_{\odot}\right)$ & 0.47 & 0.47 & 0.47 \\
$M_{\text {env }, 0}\left(M_{\odot}\right)$ & 0.005 & 0.005 & 0.005 \\
$\log g$ & 5.69 & 5.69 & 5.69 \\
$T_{\text {eff }}(\mathrm{K})$ & 33558 & 33558 & 33558 \\
EHB age $(\mathrm{yr})$ & $1.8 \times 10^{8}$ & $1.8 \times 10^{8}$ & $1.8 \times 10^{8}$ \\
\hline
\end{tabular}

these are not available yet. We are currently developing a similar approach to the one presented here to study the long-period sdB pulsators.

Acknowledgements. We are grateful to P. P. Eggleton for the use of his evolution code, and to E. Glebbeek and S. de Mink for their help with this code. We thank W. van Ham for his help with the frequency-matching algorithm. We would also like to thank M. Vučković, R. Østensen, and M. D. Reed for stimulating discussions. We also thank the referee, Z. Han, for useful comments. H.H. thanks the department of Astrophysics at the University of Liège for its hospitality. H.H. acknowledges a Ph.D. scholarship through the "Convenant Katholieke Universiteit Leuven, Belgium - Radboud Universiteit Nijmegen, the Netherlands". C.A. acknowledges financial support from the "Stichting Nijmeegs UniversiteitsFonds (SNUF)" and The Netherlands Research School for Astronomy (NOVA). H.H. and C.A. are supported by the Research Council of Leuven University, through grant GOA/2003/04. G.N. is supported by NWO-VENI grant 639.041.405. This work was supported by the European Helio- and Asteroseismology Network (HELAS), a major international collaboration funded by the European Commission's Sixth Framework Programme.

\section{References}

Aerts, C., Thoul, A., Daszyńska, J., et al. 2003, Science, 300, 1926

Allard, F., Wesemael, F., Fontaine, G., Bergeron, P., \& Lamontagne, R. 1994, AJ, 107, 1565

Badnell, N. R., \& Seaton, M. J. 2003, J. Phys. B Atom. Mol. Phys., 36, 4367

Brassard, P., Fontaine, G., Billères, M., et al. 2001, ApJ, 563, 1013

Brown, T. M., Sweigart, A. V., Lanz, T., Landsman, W. B., \& Hubeny, I. 2001, ApJ, 562, 368

Canuto, V. 1970, ApJ, 159, 641

Carraro, G., Girardi, L., Bressan, A., \& Chiosi, C. 1996, A\&A, 305, 849

Cassisi, S., Schlattl, H., Salaris, M., \& Weiss, A. 2003, ApJ, 582, L43

Castellani, M., \& Castellani, V. 1993, ApJ, 407, 649

Caughlan, G. R., \& Fowler, W. A. 1988, Atomic Data and Nuclear Data Tables, 40,283

Caughlan, G. R., Fowler, W. A., Harris, M. J., \& Zimmerman, B. A. 1985, Atomic Data and Nuclear Data Tables, 32, 197

Charpinet, S., Fontaine, G., Brassard, P., \& Dorman, B. 1996, ApJ, 471, L103

Charpinet, S., Fontaine, G., Brassard, P., et al. 1997, ApJ, 483, L123

Charpinet, S., Fontaine, G., Brassard, P., \& Dorman, B. 2000, ApJS, 131, 223

Chayer, P., Fontaine, G., \& Wesemael, F. 1995, ApJS, 99, 189

D’Cruz, N. L., Dorman, B., Rood, R. T., \& O’Connell, R. W. 1996, ApJ, 466, 359

Dupret, M. A. 2001, A\&A, 366, 166

Edelmann, H., Heber, U., Hagen, H.-J., et al. 2003, A\&A, 400, 939

Eggleton, P. P. 1971, MNRAS, 151, 351

Eggleton, P. P. 1972, MNRAS, 156, 361

Eggleton, P. P. 1973, MNRAS, 163, 279

Eggleton, P. P., Faulkner, J., \& Flannery, B. P. 1973, A\&A, 23, 325

Eldridge, J. J., \& Tout, C. A. 2004, MNRAS, 348, 201

Fontaine, G., Brassard, P., Charpinet, S., \& Chayer, P. 2006, Mem. Soc. Astron. Ital., 77, 49

Fontaine, G., Brassard, P., Charpinet, S., et al. 2003, ApJ, 597, 518

Green, E. M., Fontaine, G., Reed, M. D., et al. 2003, ApJ, 583, L31

Han, Z., Podsiadlowski, P., \& Eggleton, P. P. 1994, MNRAS, 270, 121 
Han, Z., Podsiadlowski, P., Maxted, P. F. L., Marsh, T. R., \& Ivanova, N. 2002, MNRAS, 336, 449

Han, Z., Podsiadlowski, P., Maxted, P. F. L., \& Marsh, T. R. 2003, MNRAS, 341, 669

Heber, U. 1986, A\&A, 155, 33

Hu, H., Nelemans, G., Østensen, R., et al. 2007, A\&A, 473, 569

Hubbard, W. B., \& Lampe, M. 1969, ApJS, 18, 297

Iben, Jr., I. 1975, ApJ, 196, 525

Iben, Jr., I., \& Renzini, A. 1983, ARA\&A, 21, 271

Iglesias, C. A., \& Rogers, F. J. 1996, ApJ, 464, 943

Itoh, N., Adachi, T., Nakagawa, M., Kohyama, Y., \& Munakata, H. 1989, ApJ, 339,354

Itoh, N., Mutoh, H., Hikita, A., \& Kohyama, Y. 1992, ApJ, 395, 622

Jeffery, C. S., \& Saio, H. 2006, MNRAS, 372, L48

Kilkenny, D., Koen, C., O’Donoghue, D., \& Stobie, R. S. 1997, MNRAS, 285, 640

Ledoux, W. P. 1947, ApJ, 105, 305

Lisker, T., Heber, U., Napiwotzki, R., et al. 2004, Ap\&SS, 291, 351

Maxted, P. F. L., Heber, U., Marsh, T. R., \& North, R. C. 2001, MNRAS, 326, 1391

Michaud, G., Bergeron, P., Wesemael, F., \& Heber, U. 1989, ApJ, 338, 417

Michaud, G., Richer, J., \& Richard, O. 2007, ApJ, 670, 1178
Miglio, A., Bourge, P.-O., Montalbán, J., \& Dupret, M.-A. 2007, Commun. Asteroseismol., 150, 209

Morales-Rueda, L., Maxted, P. F. L., Marsh, T. R., Kilkenny, D., \& O’Donoghue, D. 2006, Baltic Astron., 15, 187

Nelemans, G., Verbunt, F., Yungelson, L. R., \& Portegies Zwart, S. F. 2000, A\&A, 360, 1011

Piersanti, L., Tornambé, A., \& Castellani, V. 2004, MNRAS, 353, 243

Pols, O. R., Schroder, K.-P., Hurley, J. R., Tout, C. A., \& Eggleton, P. P. 1998, MNRAS, 298, 525

Pols, O. R., Tout, C. A., Eggleton, P. P., \& Han, Z. 1995, MNRAS, 274, 964

Reimers, D. 1975, Mem. Soc. R. Sci. Liège, 8, 369

Richard, O., Michaud, G., \& Richer, J. 2002, ApJ, 580, 1100

Saffer, R. A., Bergeron, P., Koester, D., \& Liebert, J. 1994, ApJ, 432, 351

Schlattl, H., Cassisi, S., Salaris, M., \& Weiss, A. 2001, ApJ, 559, 1082

Schröder, K.-P., Pols, O. R., \& Eggleton, P. P. 1997, MNRAS, 285, 696

Scuflaire, R., Montalbán, J., Théado, S., et al. 2007, Ap\&SS, 322

Seaton, M. J., \& Badnell, N. R. 2004, MNRAS, 354, 457

Seaton, M. J., Yan, Y., Mihalas, D., \& Pradhan, A. K. 1994, MNRAS, 266, 805

Serenelli, A., \& Weiss, A. 2005, A\&A, 442, 1041

Unglaub, K., \& Bues, I. 2001, A\&A, 374, 570

Vink, J. S., \& Cassisi, S. 2002, A\&A, 392, 553

Vučković, M., Aerts, C., Östensen, R., et al. 2007, A\&A, 471, 605 